\title{
Electrical conductivity of ferroelectric sodium vanadate, rubidium vanadate, cesium vanadate and their solid solutions
}

\author{
V V PATIL, A P KASHID ${ }^{1}$ and S H CHAVAN*2 \\ Department of Physics, Main Rajaram Jr. College, Kolhapur 416 002, India \\ ${ }^{1}$ Warana Mahavidyalaya, Warananagar 416113 , India \\ ${ }^{2}$ Department of Physics, Shivaji University, Kolhapur 416004 , India \\ MS received 29 August 1988; revised 29 April 1989
}

\begin{abstract}
The d.c. electrical conductivity of sodium vanadate, rubidium vanadate, cesium vanadate and their solid solutions sodium-rubidium vanadate and sodium-cesium vanadate were studied by a two-probe method in the temperature range covering their transition points. The electrical conductivity shows sharp change at the phase transition temperature of these materials. In $\mathrm{NaVO}_{3}, \mathrm{RbVO}_{3}$ and $\mathrm{CsVO}_{3}$, increase in d.c. conductivity is observed in the ferroelectric region while nonlinearities are observed above transition temperatures. In solid solutions, the activation energy in the paraelectric state is higher than that in the ferroelectric state and depends upon sodium concentration.
\end{abstract}

Keywords. Ferroelectrics; conductivity; phase transition; activation energy; critical field.

\section{Introduction}

Nearly all ferroelectric materials which have high Curie temperatures lose their piezoelectric properties at temperatures below their Curie points according to Grawford (1959). It was reported by Guruvich and $\operatorname{Rez}(1960)$ that the loss of piezoelectric properties was due to increase in the electrical conductivity of these materials at high temperatures. Verma and Lal (1981) reported the electrical transport on heavy rare-earth tungstates and found that these materials are mixed ionic-electronic conductors. Lal et al (1982) reported the electrical transport in heavy rare-earth iron garnets. Tripathi and Lal (1982) studied the electric transport behaviour of some light rare-earth orthochromites. Patil et al (1987) studied the electrical conductivity of the ferroelectric vanadates of sodium, potassium and lithium and have also reported the Curie temperature of lithium, potassium and rubidium vanadate as $410^{\circ} \mathrm{C}, 318^{\circ} \mathrm{C}$ and $372^{\circ} \mathrm{C}$ respectively in dielectric studies. Sawada and Nomura (1951) reported that $\mathrm{NaVO}_{3}$ is ferroelectric below $380^{\circ} \mathrm{C}$.

This paper reports the variation of d.c. electrical conductivity with temperature of sodium vanadate $\left(\mathrm{NaVO}_{3}\right)$, rubidium vanadate $\left(\mathrm{RbVO}_{3}\right)$, cesium vanadate $\left(\mathrm{CsVO}_{3}\right)$ and their solid solutions sodium-rubidium vanadate $\left(\mathrm{Na}_{x}-\mathrm{Rb}_{1-x}\right) \mathrm{VO}_{3}$ and sodium-cesium vanadate $\left(\mathrm{Na}_{x}-\mathrm{Cs}_{1-x}\right) \mathrm{VO}_{3}$.

\section{Experimental}

Sodium vanadate ceramics were prepared from a mixture of sodium carbonate and vanadium pentoxide in 1:1 molar proportions, heated in a platinum crucible in a globar furnace up to $750^{\circ} \mathrm{C}$ for $4 \mathrm{~h}$. $\mathrm{RbVO}_{3}$ and $\mathrm{CsVO}_{3}$ ceramics were similarly prepared.

\footnotetext{
*For correspondence.
} 
Solid solutions $\left(\mathrm{Na}_{x}-\mathrm{Rb}_{1-x}\right) \mathrm{VO}_{3}$ were prepared from a mixture of $\mathrm{NaVO}_{3}$ and $\mathrm{RbVO}_{3}$ having molar proportions $(x=0.75,0.50,0.25)$, and heated in a globar furnace at $750^{\circ} \mathrm{C}$ for $4 \mathrm{~h}$ in a platinum crucible. The solid solutions of $\left(\mathrm{Na}_{x^{-}}\right.$ $\left.\mathrm{Cs}_{1-x}\right) \mathrm{VO}_{3}(x=0.75,0.50,0.25)$ were prepared by the same method. The prepared samples were confirmed by use of an X-ray diffractometer. The pellets of these materials having thicknesses of about $1 \mathrm{~mm}$ and diameters of $1 \mathrm{~cm}$ were prepared under a pressure of 5 tonnes by using a hydraulic press. The test samples were sintered at $350^{\circ} \mathrm{C}$ for $3 \mathrm{~h}$. For good thermal contact the two faces of these test samples were painted with silver paste and were placed in the sample holder with stainless-steel electrodes fabricated in our laboratory.

The experimental set-up consists of a globar furnace, a transistorised power supply unit to provide d.c. electric field, a digital micro-voltmeter (VMV 15), a micro-ampere current meter, a digital multimeter and a temperature controller arrangement. Current through the pellets was measured as a function of applied d.c. electric field at a constant temperature and the current density $J$ was plotted against the applied electric field $(E)$. Three such plots are shown in figure 1. The current density $J$ varies linearly with $E$ in accordance with Ohm's law up to the critical field, the variation then becomes nonlinear. For the further study of d.c. conductivity, the value of d.c. electric field $E$ chosen is $14 \mathrm{~V}$, which is within the critical field. The d.c. conductivity measurements were based on the d.c. resistivity data obtained by the two-probe method in the temperature range covering their transition points for the above materials. The d.c. conductivities were obtained from resistivity data. A d.c. voltage of $14 \mathrm{~V}$ was applied across the pellet holder in series with a pico-ammeter and the sample was heated slowly in a furnace. In order to

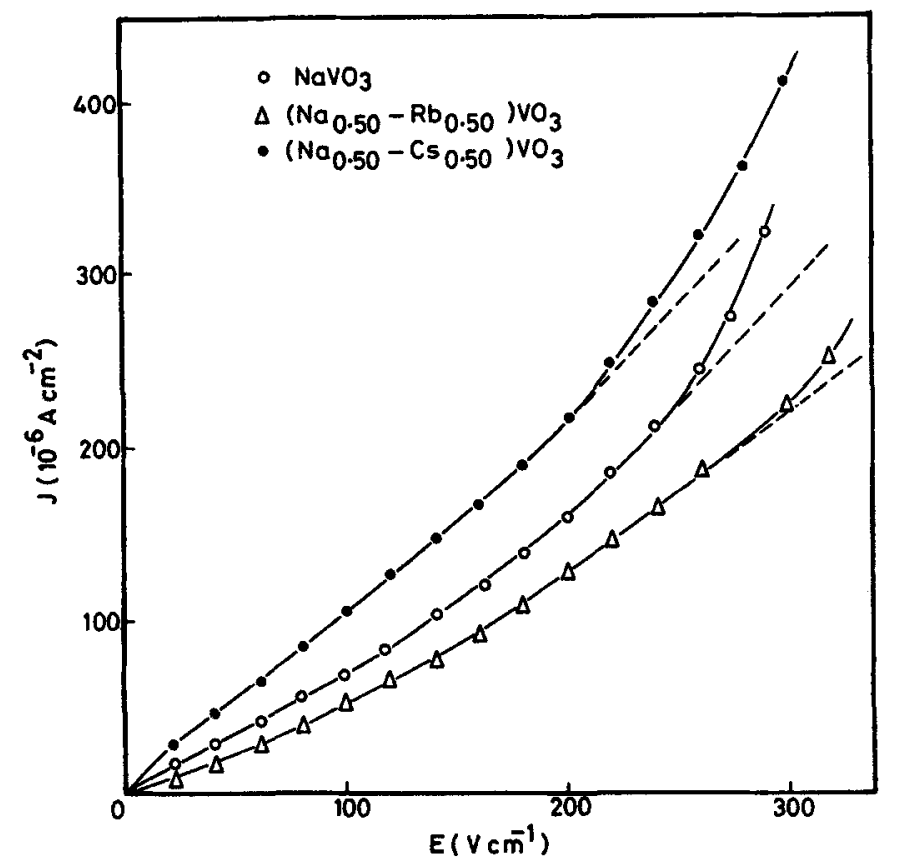

Figure 1. Variation of current density $J$ with d.c. electric field $E$ [Temperature for $\left.\mathrm{NaVO}_{3}=325^{\circ} \mathrm{C},\left(\mathrm{Na}_{0.50^{0}}-\mathrm{Rb}_{0.50}\right) \mathrm{VO}_{3}=310^{\circ} \mathrm{C},\left(\mathrm{Na}_{0.50}-\mathrm{Cs}_{0.50}\right) \mathrm{VO}_{3}=325^{\circ} \mathrm{C}\right]$. 
study the nature of conductivity the graph of $\log \sigma$ with $\log t$ at various constant temperatures are plotted. The overall accuracy and reproducibility in the measurement of the $T_{t r}$ is about $1 \%$ at all temperatures.

\section{Results and discussion}

The results of measurements of electrical conductivity in $\mathrm{NaVO}_{3}, \mathrm{RbVO}_{3}, \mathrm{CsVO}_{3}$ and their solid solutions $\left(\mathrm{Na}_{x}-\mathrm{Rb}_{1-x}\right) \mathrm{VO}_{3},\left(\mathrm{Na}_{x}-\mathrm{Cs}_{1-x}\right) \mathrm{VO}_{3}$ obey the conventional exponential relation,

$$
\sigma=\sigma_{0} \exp (-\Delta E / k T)
$$

$\Delta E$ is the activation energy, $\sigma_{0}$ a constant and $k$ the Boltzmann constant. The temperature variation of $\log \sigma$ with $1 / T$ for the above materials is shown in figures 2 and 3 respectively. To study the nature of conductivity (ionic, electronic or mixed) the variation of d.c. conductivity with time is studied at constant temperature using electrodes blocking ionic conduction. For pure electronic conduction the conductivity is independent of time while it decreases with time and tends to zero after a long time for pure ionic conductors. For mixed ionic-electronic conduction d.c. conductivity initially decreases with time and then remains constant at some finite value. The electrical conductivity is due to migration of ions or electrons or both. In electrical conductivity the nature of the charge carrier plays an important role. The plot of $\log \sigma$ as a function of $\log t$ decides the nature of conduction. Such plots are shown in figure 5 for $\mathrm{NaVO}_{3}, \mathrm{RbVO}_{3}, \mathrm{CsVO}_{3},\left(\mathrm{Na}_{0.50}-\mathrm{Rb}_{0.50}\right) \mathrm{VO}_{3}$ and $\left(\mathrm{Na}_{0.50}-\mathrm{Cs}_{0.50}\right) \mathrm{VO}_{3}$ at constant temperatures. In the case of mixed ionic conductors, current continuously decreases and attains a constant value after a long

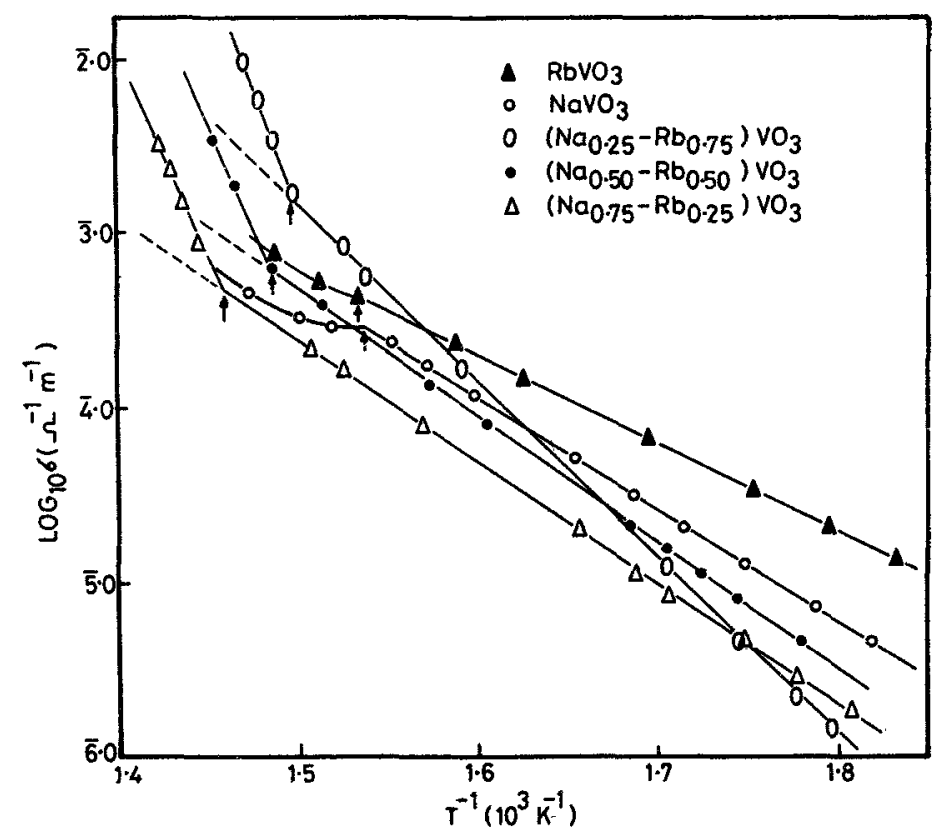

Figure 2. Variation of logarithmic conductivity $\Theta$ with $1 / T$ for $\left(\mathrm{Na}_{x}-\mathrm{Rb}_{1-x}\right) \mathrm{VO}_{3}$ 


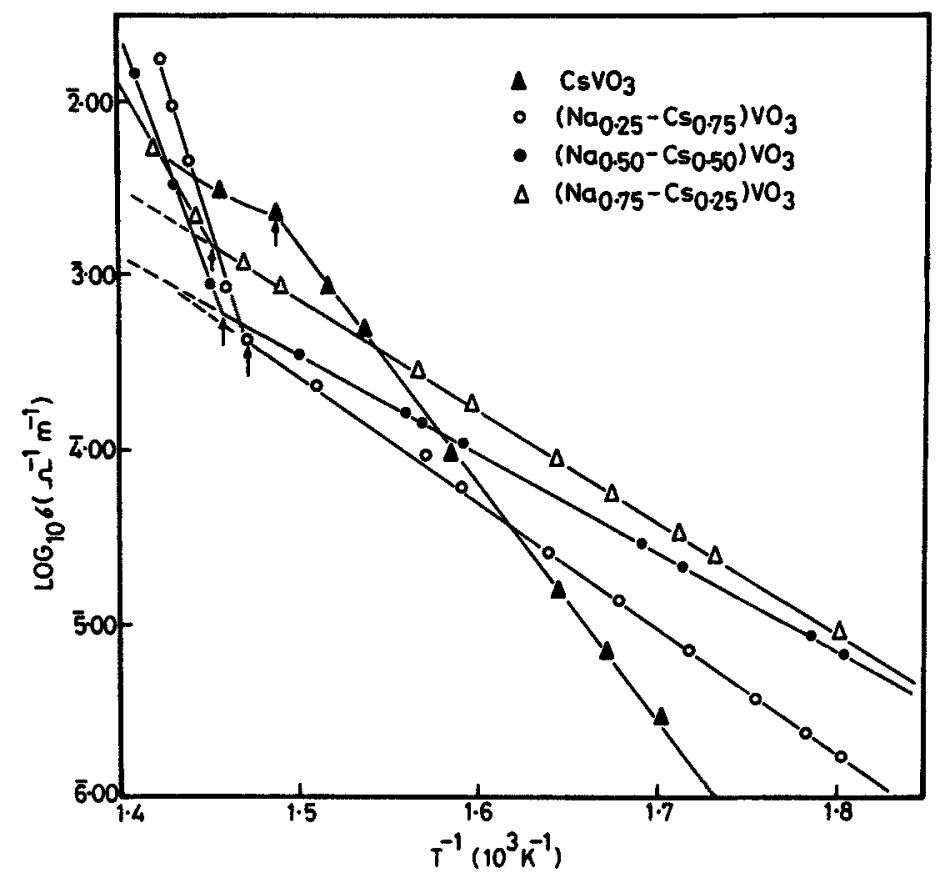

Figure 3. Variation of logarithmic conductivity $\bigcirc$ with $1 / T$ for $\left(\mathrm{Na}_{x}-\mathrm{Cs}_{1-x}\right) \mathrm{VO}_{3}$.

time. In solids with permanent dipoles a little time dependent current is expected because on the application of an electric field these dipoles tend to orient themselves in the direction of the applied field. However, it should be noted that these dipoles once aligned in the direction of applied field do not contribute to the d.c. current.

It is clear from figures 2 and 3 that the d.c. electrical conductivity for solid solutions depends exponentially on temperature. In case of $\mathrm{NaVO}_{3}, \mathrm{RbVO}_{3}$ and $\mathrm{CsVO}_{3}$ the d.c. conductivity increases exponentially only up to the transition temperature and becomes nonlinear above the Curie temperature. For solid solutions a sharp change in d.c. conductivity is observed at the Curie temperatures. These temperatures are ferroelectric Curie temperatures of the respective samples which were investigated by the hysteresis loop-method (Sawyer and Tower 1930) shown in table 1 . It is seen from figure 4 that the conductivity for $\mathrm{NaVO}_{3}, \mathrm{RbVO}_{3}$, $\mathrm{CsVO}_{3},\left(\mathrm{Na}_{0.50}-\mathrm{Rb}_{0.50}\right) \mathrm{VO}_{3}$ and $\left(\mathrm{Na}_{0.50}-\mathrm{Cs}_{0.50}\right) \mathrm{VO}_{3}$ below transition temperature is of the mixed type (ionic-electronic).

From the slopes of plots of $\log \sigma$ vs. $1 / T$, the activation energies are calculated both in ferroelectric and paraelectric states for the above materials and are summarized in table 1. Table 1 reveals that the activation energy for solid solutions in the ferroelectric state is less than that in paraelectric state which is in agreement with the results obtained by Patil et al (1987), for $\mathrm{NaVO}_{3}, \mathrm{KVO}_{3}, \mathrm{LiVO}_{3}$ and their solid solutions.

\section{Conclusions}

(i) For $\mathrm{NaVO}_{3}, \mathrm{RbVO}_{3}, \mathrm{CsVO}_{3}$, the d.c. electrical conductivity increases 
Table 1. Activation energies and Curie temperatures of different samples.

\begin{tabular}{lccccc}
\hline & \multicolumn{2}{c}{ Activation energy (eV) } & Curie temp. \\
\cline { 2 - 6 } Sample & Ferro-state & Para-state & $\begin{array}{c}T_{r \text { rr }}\left({ }^{\circ} \mathrm{C}\right) \text { by } \\
\text { hysteresis }\end{array}$ & $\begin{array}{c}T_{r}\left({ }^{\circ} \mathrm{C}\right) \text { by } \\
\text { pyroelectric } \\
\text { measurement }\end{array}$ \\
\hline $\mathrm{NaVO}_{3}$ & 1.17 & - & 375 & 375 & 375 \\
$\mathrm{RbVO}_{3}$ & 0.99 & - & 372 & 370 & 370 \\
$\mathrm{Cs} \mathrm{VO}_{3}$ & 2.83 & - & 400 & 400 & 395 \\
$\left(\mathrm{Na}_{0.75}-\mathrm{Rb}_{\mathbf{0} .25}\right) \mathrm{VO}_{3}$ & 1.32 & 5.2 & 412 & 410 & 410 \\
$\left(\mathrm{Na}_{0.50}-\mathrm{Rb}_{0.50}\right) \mathrm{VO}_{3}$ & 1.39 & 4.82 & 398 & 400 & 400 \\
$\left(\mathrm{Na}_{0.25}-\mathrm{Rb}_{0.75}\right) \mathrm{VO}_{3}$ & 1.98 & 4.62 & 393 & 390 & 390 \\
$\left(\mathrm{Na}_{0.75}-\mathrm{Cs}_{0.25}\right) \mathrm{VO}_{3}$ & 1.32 & 3.57 & 416 & 415 & 415 \\
$\left(\mathrm{Na}_{0.50}-\mathrm{Cs}_{0.50}\right) \mathrm{VO}_{3}$ & 1.34 & 5.67 & 412 & 410 & 410 \\
$\left(\mathrm{Na}_{0.25}-\mathrm{Cs}_{0.75}\right) \mathrm{VO}_{3}$ & 1.45 & 6.32 & 407 & 405 & 405 \\
\hline
\end{tabular}

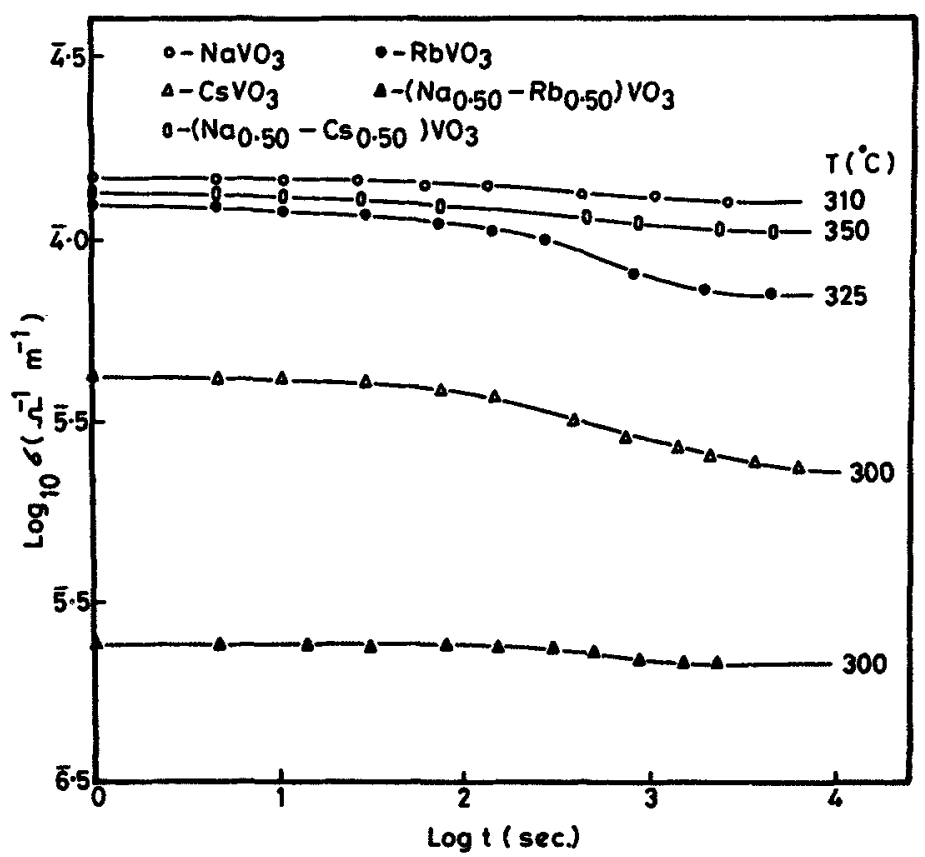

Figure 4. Variation of logarithmic conductivity $O$ with $\log t$ for $\mathrm{NaVO}_{3}, \mathrm{RbVO}_{3}, \mathrm{CsVO}_{3}$, $\left(\mathrm{Na}_{0.50}-\mathrm{Rb}_{0.50}\right) \mathrm{VO}_{3}$ and $\left(\mathrm{Na}_{0.50}-\mathrm{Cs}_{0.50}\right) \mathrm{VO}_{3}$.

ixponentially in the ferroelectric region while nonlinearities are observed in the paraelectric state.

(ii) The nature of electrical conductivity of these materials is of a mixed type in the ferroelectric region.

(iii) A sharp change in electrical conductivity is observed at the phase transition temperatures which are the ferroelectric Curie temperatures of the respective materials.

(iv) The activation energy of the solid solutions is higher in the paraelectric region than in the ferroelectric region and it depends upon sodium concentration. 


\section{References}

Grawford A $1959 \mathrm{Br}$. Commun. Electron. 6516

Guruvich V and Rez 11960 Sot. Phys. Solid State (Engl. Transl.) 2624

Lal H, Verma B and Yadav V 1982 J. Mater. Sci. 173317

Palil T A. Jamadar V M and Chavan S H 1987a Bull. Mater. Sci. 9331

Patil T A, Jamadar V M and Chavan S H 1987b Indian J. Phys. A62 87

Salwada $S$ and Nomura $S 1951$ J. Phys. Soc. Jpn. 6.192

Sawyer C B and Tower C H 1930 Phys. Ret. (USA) 35269

Tripathi A K and Lal H B 1982 J. Matter. Sci. 171595

Verma B and Lal H 1981 Mater. Res. Bull. 161579 